\title{
Brauchen versus kaufen: Wenn Warenkonsum zur Sucht wird
}

\author{
Nora M. Laskowskia ${ }^{a}$ Patrick Trotzke ${ }^{b} \quad$ Astrid Müller ${ }^{a}$ \\ ${ }^{a}$ Klinik für Psychosomatik und Psychotherapie, Medizinische Hochschule Hannover, Hannover, Deutschland; \\ ${ }^{\mathrm{b}}$ Center for Behavioral Addiction Research, Allgemeine Psychologie: Kognition, Universität Duisburg-Essen, Duisburg, Deutschland
}

\section{Schlüsselwörter}

Pathologisches Kaufen · Kaufsucht · «Buying-shopping disorder" - "Compulsive buying" · Verhaltenssucht

\section{Zusammenfassung}

Obwohl pathologisches Kaufen ein seit mehr als 100 Jahren bekanntes Phänomen darstellt, ist das Störungsbild noch nicht als eigenständige Erkrankung anerkannt. Laut einer Meta-Analyse zeigen 5\% der erwachsenen Allgemeinbevölkerung eine Neigung zu pathologischem Kaufen. Das Störungsbild ist geprägt von einem unwiderstehlichen Kaufdrang, einer immensen gedanklichen Beschäftigung mit Kaufen sowie unangemessenen Kaufepisoden, die der Emotionsregulation dienen. Die erworbenen Konsumgüter werden in der Regel kaum oder gar nicht benutzt. Betroffene und Angehörige erleben einen immensen Leidensdruck, der aus sozialen, beruflichen, psychischen und rechtlichen Folgeproblemen resultiert. Psychische Komorbiditäten sind typischerweise depressive und Angststörungen, pathologisches Horten, Binge-Eating-Störung, Bulimia nervosa, Glücksspielstörung und Substanzkonsumstörungen. Experimentelle Studien legen nahe, pathologisches Kaufen als eine "Störung in Zusammenhang mit süchtigem Verhalten» (Verhaltenssucht) zu klassifizieren.

(C) 2018 S. Karger GmbH, Freiburg

Aus Gründen der Lesbarkeit wird in diesem Artikel der generische Maskulin verwendet, wobei selbstverständlich immer beide Geschlechter gleichermaßen gemeint sind.

\section{Keywords}

Pathological buying - Shopping addiction . Buying-shopping disorder . Compulsive buying . Behavioral addiction

\section{Summary}

Needing versus Buying: When Excessive Consumption of Goods Becomes an Addiction

Although pathological buying was first described over 100 years ago, it is still not recognized as an independent mental disorder. This is in contrast to a recent metaanalysis indicating an estimated point prevalence of $5 \%$ for pathological buying. Pathological buying is characterized by a strong urge to buy, an extreme preoccupation with buying and shopping, and maladaptive buying episodes in order to regulate emotions. The acquired goods are rarely or not at all used. The recurrent excessive-buying episodes lead to severe distress due to social, occupational, psychological, and legal consequences. Common comorbid psychiatric disorders are depressive and anxiety disorders, hoarding disorder, binge eating disorder, bulimia nervosa, gambling disorder, and substance use disorders. Recent research findings indicate that pathological buying should be considered a behavioral addiction and classified as a 'disorder due to addictive behaviors'.

\section{KARGER} ๑ 2018 S. Karger GmbH, Freiburg 


\section{Einleitung}

Bereits vor mehr als 100 Jahren wurde pathologisches Kaufen in der Fachliteratur erwähnt [Kraepelin, 1909]. Kraepelin beschrieb, dass Menschen mit «...krankhafte[r] Kauflust...» [Kraepelin, 1909] bedarfsunabhängig und in einer hohen Stückzahl Waren konsumieren, sobald die Möglichkeit dazu besteht. Auch Bleuler berichtete 1923 über das Krankheitsbild und schrieb, dass die Betroffenen einen starken Kaufdrang spüren, dem sie kaum etwas entgegensetzen können [Bleuler, 1923]. Das Phänomen «pathologisches Kaufen» ist demnach nicht ausschließlich auf die neuzeitliche Konsumentwicklung in unserer gegenwärtigen Gesellschaft zurückzuführen. Obwohl die Klassifikation von pathologischem Kaufen seit mindestens 30 Jahren ernsthaft diskutiert wird, konnte das Krankheitsbild in der 5. Auflage des «Diagnostic and Statistical Manual of Mental Disorders» (DSM-5) [APA, 2015] nicht als eigenständige Erkrankung verschlüsselt werden und wird es vermutlich auch nicht in der 11. Revision der «Internationalen statistischen Klassifikation der Krankheiten und verwandter Gesundheitsprobleme» (ICD-11) [WHO, 2018]. Aufgrund der fehlenden nosologischen Einordnung sind auch die Begriffe in der Fachliteratur sehr unterschiedlich. Kraepelin führte 1909 den Begriff «Oniomanie» ein [Kraepelin, 1909], der sich aus den griechischen Wortbestandteilen «Kaufen» und «Leidenschaft/Trieb/Zwang» zusammensetzt, jedoch nur vereinzelt übernommen wurde. Im deutschen Sprachraum sind, wie auch in diesem Artikel, die Begriffe «Kaufsucht» und «pathologisches Kaufen» am häufigsten in Gebrauch.

Das Ziel dieser Arbeit ist es, basierend auf einer narrativen Literaturübersicht sowie eigenen klinischen Erfahrungen und Studienergebnissen über pathologisches Kaufen zu informieren. Nach einer ausführlichen Beschreibung des Störungsbilds befasst sich der zweite Teil dieser Arbeit vor allem mit aktuellen Überlegungen zur Klassifikation desselben.

\section{Beschreibung des Störungsbilds}

\section{Phänomenologie}

Charakteristisch für pathologisches Kaufen sind ein als unwiderstehlich wahrgenommener Kaufdrang, eine starke gedankliche Beschäftigung mit Warenkonsum und ein wiederholter Kontrollverlust beim Einkaufen, was in exzessiven, unangemessenen Kaufattacken resultiert. Dabei werden Waren konsumiert, die in ihrer Art oder Anzahl nicht benötigt werden [McElroy et al., 1994]. Dies verdeutlicht, dass für Menschen mit pathologischem Kaufen weniger der nachhaltige Gebrauch der Konsumgüter im Vordergrund steht, sondern eher der belohnende oder entlastende und oft auch identitätsstiftende Kaufakt an sich [Dittmar, 2005b; McElroy et al., 1994; Miltenberger et al., 2003]. Dafür spricht auch, dass die Waren wenig oder nicht genutzt und teilweise gar nicht erst ausgepackt werden. Bei einigen Patienten ist zudem das pathologische Horten dieser Waren zu beobachten [Müller et al., 2007]. Langfristig führen die regelmäßig auftretenden unangemessenen Kaufepisoden zu einem enormen Leidensdruck, der durch finanzielle, sozi- ale oder berufliche Probleme sowie eine reduzierte Lebensqualität hervorgerufen werden kann [Williams, 2012; Zhang et al., 2017]. Häufig werden Waren gekauft, die die finanziellen Ressourcen der Betroffenen übersteigen, und einige Patienten berichten aus diesem Grund auch von Straftaten, z.B. einem Eingehungsbetrug, die sie begehen, um ihren Kaufdrang trotz Geldnot zu befriedigen. Im sozialen Umfeld zeigen sich die Probleme häufig in Form von Lügen bezüglich des Kaufverhaltens, dem Verstecken und Verheimlichen der gekauften Waren oder dem Betrügen des familiären bzw. sozialen Umfelds.

Zu Beginn der Erkrankung überwiegen die positiven Gefühle beim und nach dem Kaufen. Im Verlauf werden die Kaufepisoden zunehmend durch negative Stimmungen oder Gefühle ausgelöst und dazu genutzt, negative Empfindungen zu überdecken, Stresserleben zu regulieren, sich von Problemen abzulenken oder sich selbstbewusster zu fühlen [Kyrios et al., 2013; Miltenberger et al., 2003; Müller et al., 2012]. Die durch den Kauf induzierte Stimmungsbesserung ist allerdings nicht von langer Dauer, da in vielen Fällen bereits kurz nach dem Kauf Schamgefühle und ein schlechtes Gewissen einsetzen [McElroy et al., 1994]. Während der Kaufepisoden werden die langfristigen negativen Folgen häufig ausgeblendet, weswegen der inadäquate Konsum trotz erheblicher aversiver Konsequenzen von den Betroffenen nicht gestoppt wird. Menschen mit pathologischem Kaufen bagatellisieren bzw. rationalisieren ihr Kaufverhalten oft. Dabei verursachen die wahrgenommene Unangemessenheit des eigenen Warenkonsums sowie das Unbehagen bezüglich des wiederholten Kontrollverlusts durchaus einen enormen Leidensdruck [McElroy et al., 1994] und beschränken die Lebensqualität der Betroffenen [Williams, 2012; Zhang et al., 2017].

Wie bereits erwähnt stellen der unwiderstehliche Kaufdrang und die massive gedankliche Beschäftigung der Betroffenen mit dem Thema «Kaufen» (im englischen Sprachraum als «preoccupation» bezeichnet) wesentliche Aspekte der Phänomenologie von pathologischem Kaufen dar [McElroy et al., 1994]. Viele Patienten berichten von dem Bedürfnis, stundenlang auf Kaufportalen im Internet $\mathrm{zu}$ «browsen» und Waren in den Bestellkorb zu legen, sowie von ständigen Gedanken an bestimmte Konsumgüter, von denen sie sich schwer ablenken können. Auch bei Patienten, die kaum noch über Geldressourcen verfügen (z.B. aufgrund einer Privatinsolvenz) und die keine Einkäufe mehr tätigen, sind «windowshopping», «browsing» und eine starke gedankliche Beschäftigung mit Kaufaspekten zu beobachten. Zudem werden von vielen Patienten Entzugserscheinungen beschrieben (z.B. Zittern, Schwitzen, Dysphorie). Diese können auftreten, wenn sie am Kaufen gehindert werden oder sich selbst einschränken. Auch Toleranzentwicklungen werden erwähnt. So können z.B. immer häufigere oder immer teurere Käufe nötig werden, um die gleiche Befriedigung zu spüren. In Kasten 1 wird anhand von 2 Fallbeispielen die Phänomenologie von pathologischem Kaufen illustriert.

Die Gruppe der Menschen mit pathologischem Kaufen ist in ihrem Verhalten sehr heterogen. Sowohl die gekauften Waren als auch der Ort des Konsums richten sich nach persönlichen Präferenzen. Einige Patienten kaufen bevorzugt in Kaufhäusern oder 
Frau B. berichtet, dass sie seit etwa 40 Jahren an pathologischem Kaufen leide. Wenn sie Geld habe, müsse sie dieses ausgeben. Auch aktuell gehe sie bis zu 2-mal pro Woche in die Stadt und kaufe Dinge ein, die sie nicht benötige. Es handele sich um Einkäufe in Spezialgeschäften von oft teuren, hochwertigen Waren, nicht um Internet-Shopping. Sie berichtet von ständigen Gedanken ans Kaufen. Vor dem Kauf empfinde sie Vorfreude und sei aufgeregt, auch ein Hochgefühl beschreibt sie. Nach dem Kauf würden Scham und ein schlechtes Gewissen auftreten. Sie schaffe es nie, die Waren zurückzugeben. Frau B. belaste es sehr, dass sie bezüglich ihres Kaufverhaltens oft lüge, es vertusche oder verheimliche und z.B. die meisten erworbenen Waren nicht anziehe oder benutze, weil es ihr peinlich sei, so viel zu shoppen. Mittlerweile verspüre sie beim Kaufen kaum noch Vergnügen; allerdings verschaffe es ihr Entlastung, Ablenkung und Entspannung. Inzwischen habe sie aufgrund des unangemessenen Kaufverhaltens Schulden im 5-stelligen Bereich, und trotzdem gebe sie regelmäßig ihr Haushaltsgeld für unnütze Dinge aus. Bei Geldnot habe sie sogar schon das Sparbuch ihres minderjährigen Sohnes ohne dessen Wissen belastet, um sich etwas kaufen zu können. Der Hang zum Konsumieren habe im 14. Lebensjahr begonnen. Sie erinnere sich an Auseinandersetzungen mit ihren Eltern, weil sie bereits als Teenager ihr ganzes Taschengeld ausgegeben habe, und an spätere Ehekonflikte aufgrund finanzieller Engpässe infolge ihrer Kaufexzesse. Im Pathological Buying Screener (PBS) [Müller et al., 2015b] erreicht Frau B. einen Summenwert von 65 (Schwellenwert für pathologisches Kaufen $>28$ ).

Herr D. beschreibt ein episodisches pathologisches Kaufverhalten. Gegenüber seiner Ehefrau verheimliche er sein unangemessenes Kaufverhalten, da er befürchte, sein Familienleben zu ruinieren. Die Einkäufe tätige er vorrangig im Internet über sein Smartphone, oft seien es auch multiple Käufe (z.B. T-Shirts in 10 verschiedenen Farben). Auslöser für die unangemessenen Kaufepisoden seien vor allem Stimmungstiefs. Das Kaufen diene ihm als Ventil bei Stresserleben oder als Belohnungsmöglichkeit nach getaner Arbeit. Viele der erworbenen Waren packe er in letzter Zeit nicht mehr aus, sondern staple sie in einem Raum in seiner Wohnung. Durch sein Kaufverhalten habe er sich massiv verschuldet, was jedoch nicht zu einer Reduktion der Einkäufe geführt habe. Wegen Vortäuschung von Zahlungsfähigkeit und Eingehungsdelikten sei bereits eine Anzeige gegen ihn erfolgt. Gleichwohl erlebe er weiterhin folgenhafte Kontrollverluste beim Shoppen und Kaufen. Wenn er Werbung sehe, spüre er sofort einen starken Besitzwunsch, den er dann ohne nachzudenken und ohne weitere negative Konsequenzen zu antizipieren (drohende Insolvenz, Zunahme bestehender psychischer Probleme, Scheidung, Gerichtsverfahren usw.) sofort umsetze. Im PBS [Müller et al., 2015b] erreicht Herr D. einen Wert von 55 (Schwellenwert für pathologisches Kaufen > 28) und in der deutschen Shopping Version des Internet Addiction Test [Trotzke et al., 2015] einen Wert von 43 (Schwellenwert für Internet Shopping Addiction > 30).

kleinen Boutiquen ein, andere ziehen die Anonymität des Internets vor, wobei im klinischen Alltag Mischformen aus Online-Käufen und dem klassischen Erwerb in Geschäften am häufigsten zu beobachten sind. Gerade der Online-Markt vereinfacht den Konsum durch unbegrenzte Öffnungszeiten, ein großes Warenangebot und bargeldloses Bezahlen [Trotzke et al., 2015]. Frauen konsumieren eher Kleidung oder Ähnliches, während Männer häufiger zu Elektronik usw. greifen [Coley und Burgess, 2003]. Ebenfalls unterscheiden sich die Betroffenen dahingehend, ob sie eher teure Markenartikel kaufen oder auf preiswerte Sonderangebote fixiert sind. Neben dem Konsum von Dingen für sich selbst berichten viele $\mathrm{Pa}$ tienten, dass sie auch unaufgefordert Geschenke für das soziale Umfeld erwerben (z.B. für Familienangehörige, Freunde).

Der Verlauf von pathologischem Kaufen kann bisher nur anhand von Patientenschilderungen beurteilt werden, da keine Daten aus Längsschnittstudien vorliegen. Die Patienten berichten meistens chronische, episodenhafte Verläufe, die durch einen Wechsel zwischen häufigen Kaufepisoden und symptomfreien Zeiten geprägt sind. Erste entgleiste Kaufprozesse können schon im Jugendalter auftreten [Black et al., 2016]. Ein (Wieder-)Anstieg der Symptome oder des Schweregrads von pathologischem Kaufen wird anamnestisch häufig in einem zeitlichen Zusammenhang mit negativen Erlebnissen (z.B. familiäre, berufliche Belastungen) beschrieben.

\section{Psychische Komorbidität}

Patienten mit pathologischem Kaufen leiden nahezu immer an weiteren psychischen Erkrankungen, was zur Entwicklung und
Aufrechterhaltung des Störungsbilds beiträgt. Zu den häufigsten Begleiterkrankungen zählen Depressionen, soziale Phobie, pathologisches Horten (der Konsumgüter), Binge-Eating-Störung, Bulimia nervosa, Glücksspielstörung und Substanzkonsumstörungen (vor allem Alkoholkonsumstörung) [Granero et al., 2016; Müller et al., 2015a]. Die häufigsten komorbiden Persönlichkeitsstörungen sind ängstlich-vermeidende, zwanghafte und Borderline-Persönlichkeitsstörungen [Claes und Müller, 2017]. Aufgrund fehlender Längsschnittstudien kann keine Aussage zur Chronologie der jeweiligen Erkrankungen getroffen werden. Laut Patientenberichten scheinen ängstlich-depressive Symptome und Minderwertigkeitsgefühle häufig zur Entstehung von pathologischem Kaufen beizutragen. Vice versa ist oft zu beobachten, dass pathologisches Kaufverhalten zu einer Zunahme bestehender oder zum Neuauftreten von psychischen Symptomen führt. So kann beispielsweise die exzessive Akquise von Konsumgütern verbunden mit einer engen emotionalen Bindung an dieselben ein pathologisches Horten der Waren nach sich ziehen, was wiederum mit der Zunahme depressiver Symptome vergesellschaftet sein kann.

\section{Diagnostik}

Die Diagnose pathologisches Kaufen kann nur anhand eines klinischen Interviews gestellt werden. Als nützlich haben sich hierfür das Strukturierte Klinische Interviewmodul für Impulskontrollstörungen [First et al., 2007] sowie die Shopping Version der YaleBrown Obsessive Compulsive Scale (Y-BOCS) [Monahan et al., 1996] erwiesen. Beide Interviews liegen leider nicht als validierte deutsche Versionen vor. 
Tab. 1. Diagnosekriterien für «compulsive buying» nach McElroy et al. [1994]

\begin{tabular}{ll}
\hline Kriterium & Beschreibung \\
\hline A & fehlangepasste starke Beschäftigung mit Erwerben bzw. Kaufen oder fehlangepasste Erwerbs- bzw. Kaufimpulse \\
& bzw. Kaufverhaltensweisen, auf die mindestens eine der folgenden Beschreibungen passt: \\
& häufige starke Beschäftigung mit Kaufen oder Kaufimpulsen, die als unwiderstehlich, sich aufdrängend, intrusiv \\
& und/oder sinnlos wahrgenommen wird; \\
& häufiges Kaufen von mehr Dingen als man sich leisten kann / häufiges Kaufen von Dingen, die nicht benötigt \\
& werden / Kaufen über längere Zeitperioden als geplant \\
\hline B & der Kaufdrang, die Kaufimpulse oder die Kaufverhaltensweisen verursachen erhebliches Leiden; sie sind \\
& zeitaufwendig, beeinträchtigen deutlich die sozialen und beruflichen Funktionen oder haben finanzielle \\
& Probleme zur Folge (Verschuldung oder Konkurs) \\
\hline C & die exzessiven Erwerbs- oder Kaufverhaltensweisen treten nicht ausschließlich in Phasen einer Manie oder \\
& Hypomanie auf \\
\hline
\end{tabular}

Tab. 2. Der Pathological Buying Screener [Müller et al., 2015b]

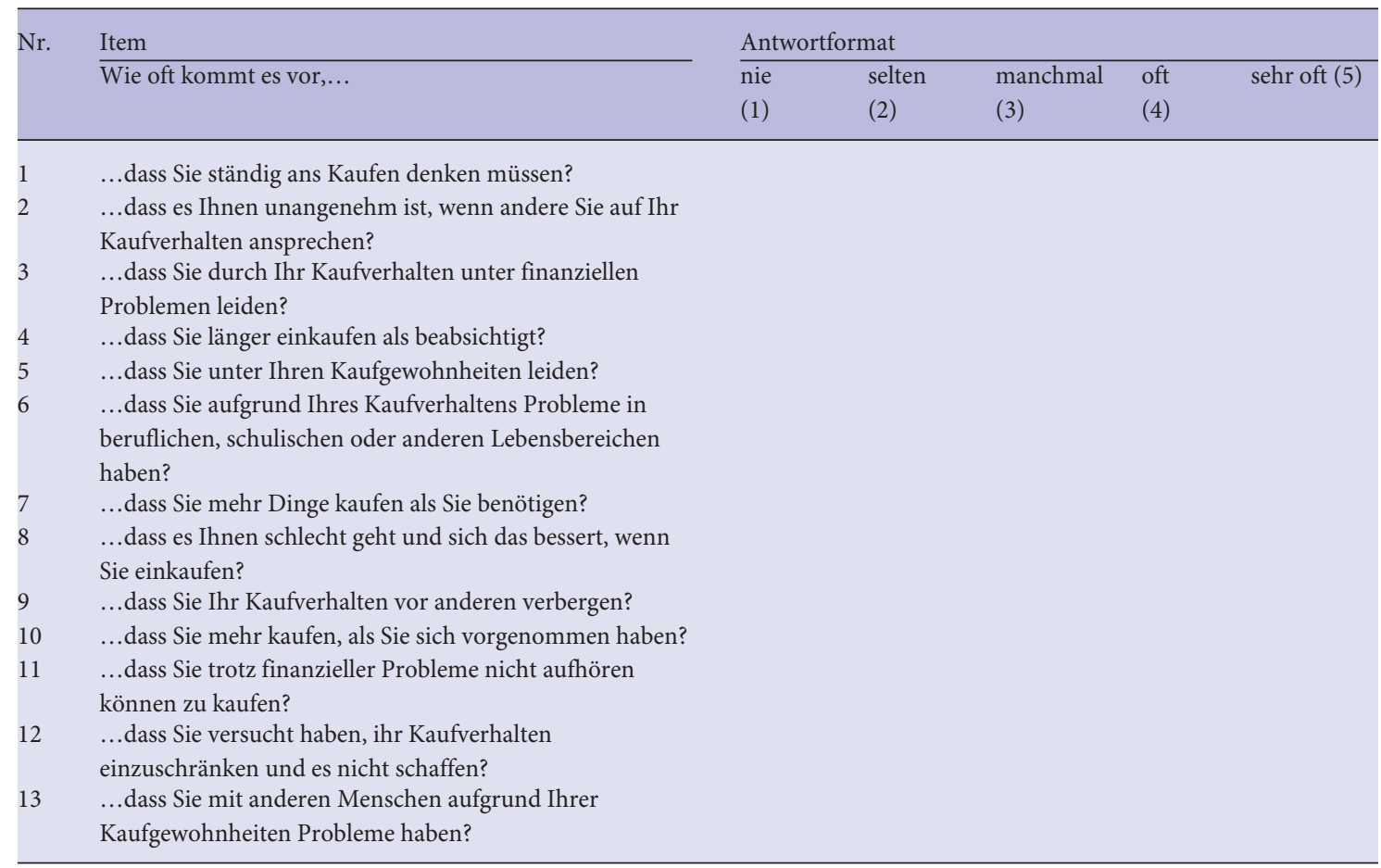

Hinsichtlich möglicher Strafdelikte (siehe oben) im Zuge eines exzessiven Warenkonsums kann unseres Erachtens nur im Rahmen eines Interviews abgeklärt werden, ob es sich um eine rein kriminelle Handlung oder um ein Betrugsdelikt im Sinne einer Beschaffungsdelinquenz im Rahmen des pathologischen Kaufens handelt. Pathologisches Kaufen ist zudem differentialdiagnostisch von der Manie abzugrenzen [McElroy et al., 1994]. Weiterhin sollte zwischen pathologischem Kaufen und seltenen impulsiven Belohnungs- oder Frustkäufen unterschieden werden. Gerade im Anfangsstadium der Erkrankung, wenn die Käufe vor allem positiv verstärkt und noch nicht habituell zur Kompensation negativer Befindlichkeiten eingesetzt werden, kann diese Unterscheidung schwerfallen. Hier empfiehlt es sich, die internen (z.B. Gedanken, Gefühle) und externen Auslöser (z.B. Werbung) sowie die treibende Motivation für die Kaufepisoden und deren Frequenz und Fol- gen genauer zu explorieren. Da bisher keine treffenderen Diagnosekriterien entwickelt wurden, wird in der Praxis häufig auf die von McElroy et al. [1994] vorgeschlagenen diagnostischen Kriterien zurückgegriffen, die jedoch bisher nicht empirisch überprüft wurden (Tab. 1).

Für eine erste Einschätzung, ob pathologisches Kaufen vorliegt, eignen sich Selbsterhebungsinstrumente in Form von Fragebögen. In deutscher Sprache liegen 3 valide und reliable Fragebögen vor: das Screeningverfahren zur Erhebung von kompensatorischem und süchtigem Kaufverhalten (SKSK) [Raab et al., 2005; Scherhorn et al., 1990], der Pathological Buying Screener (PBS) [Müller et al., 2015b] und die deutsche Version der Compulsive Buying Scale (CBS) [Müller et al., 2010]. Das SKSK wurde in den 1990er Jahren entwickelt und basiert auf dem Suchtkonzept [Raab et al., 2005; Scherhorn et al., 1990]. Es besteht aus 16 Items mit einer 4-Punkt- 
Antwortskala. Anhand des Summenwerts erlaubt das SKSK eine Kategorisierung in potenziell gefährdendes und süchtiges Kaufverhalten. Ein aktuellerer, ebenfalls in Deutschland entwickelter Fragebogen ist der bereits im Rahmen der Fallbeispiele erwähnte PBS (Tab. 2) mit 13 Items, die den folgenden 2 Subskalen zugeordnet sind: «Kontrollverlust» (10 Items) und «exzessives Kaufverhalten» (3 Items) [Müller et al., 2015b]. Zudem können 3 zusätzliche Items zur Abschätzung möglicher Symptome einer Manie/Hypomanie und pathologischen Hortens oder von Motiven der persönlichen Bereicherung ausgewertet werden [Müller et al., 2017]. Das Antwortformat ist 5-stufig. Bei der Entwicklung des Fragebogens wurden verschiedene Facetten von pathologischem Kaufen berücksichtigt: solche, die eher zum Konzept von pathologischem Kaufen als Impulskontrollstörung passen, und andere, die eher zum Verhaltenssuchtkonzept gehören (siehe unten).

Das in der Vergangenheit international meistgenutzte Selbsterhebungsinstrument ist zweifelsohne die CBS [Faber und O'Guinn, 1992], von der eine validierte deutsche Übersetzung vorliegt [Müller et al., 2010]. Sie besteht aus 7 Items, die sowohl finanzielle als auch emotionale Aspekte des pathologischen Kaufens abdecken und mit einer 5-Punkt-Likert-Skala beantwortet werden. Aufgrund der fehlenden Zeitmäßigkeit einiger Items (z.B. Item 4: «Ich habe einen Scheck ausgestellt, obwohl ich wusste, dass nicht genug Geld auf der Bank ist, um ihn zu decken» [Faber und O'Guinn, 1992]) wird diese Skala immer seltener eingesetzt.

\section{Prävalenz}

Die bisherigen Prävalenzuntersuchungen zu pathologischem Kaufen wurden mithilfe von Fragebogenerhebungen durchgeführt. Demnach kann keine genaue Prävalenz angegeben werden, es ist eher von einer Kaufsuchtgefährdung bzw. einer geschätzten Prävalenz zu sprechen.

Studien zeigen, dass pathologisches Kaufen ein internationales und kulturübergreifendes Phänomen darstellt. Eine Meta-Analyse, die Befragungen aus mehreren Ländern einbezog $(\mathrm{N}=23$ 333), ergab eine geschätzte Prävalenz von $4,9 \%$ bei Erwachsenen in der Allgemeinbevölkerung [Maraz et al., 2016]. In spezifischen Populationen, z.B. bei Studenten, liegt die angenommene Prävalenz deutlich höher (8,3\%) [Maraz et al., 2016]. In der deutschen Studie zur Validierung des oben beschriebenen PBS ( $\mathrm{N}=2403)$ zeigte sich eine geschätzte Prävalenz von 4,8\% in der Allgemeinbevölkerung [Müller et al., 2015b]. Die Neigung zu pathologischem Kaufen in Deutschland scheint seit den 1990er Jahren gestiegen zu sein, wie Konsumforscher zeigen konnten [Hubert et al., 2014; Neuner et al., 2005]. So ermittelten Neuner et al. [2005] mit dem SKSK [Raab et al., 2005] einen deutlichen Anstieg der Kaufsuchtgefährdung innerhalb von 10 Jahren in West- (Prävalenz 1991: 5,1\%; 2001: 8\%) und Ostdeutschland (Prävalanz 1991: 1\%; 2001: 6,5\%). Hubert et al. [2014] berichteten über eine weitere Zunahme von pathologischem Kaufen in Deutschland von 6,9 (2010) auf 10,4\% (2012). Auch diese Ergebnisse basieren auf Querschnittserhebungen mit dem SKSK. Mögliche Erklärungen für den Anstieg der Kaufsuchtgefährdung sind neben soziodemografischen und politischen Entwicklungen [Hubert et al., 2014; Neuner et al., 2005] auch in der
Ausweitung von pathologischem Kaufen auf den Online-Markt zu suchen [Dittmar et al., 2007]. Kritisch anzumerken ist, dass die genannten Angaben zur Zunahme von pathologischem Kaufen in der deutschen Bevölkerung auf dem Vergleich querschnittlicher Fragebogenuntersuchungen fußen und dass sie keine Prävalenz von pathologischem Kaufen abgebildet haben, sondern lediglich die Neigung hierzu in der Bevölkerung erfassen konnten. Für die Bestimmung der Prävalenz von pathologischem Kaufen bedarf es einheitlicher, anerkannter Diagnosekriterien und interviewbasierter, repräsentativer Bevölkerungserhebungen [Maraz et al., 2016].

Bezüglich eines möglichen Geschlechterunterschieds ist die Datenlage divers. Während einige Studien eine erhöhte Prävalenz bei Frauen berichteten, konnte dieser Unterschied in anderen Studien nicht festgestellt werden [Maraz et al., 2016]. Zudem verliert sich ein möglicher Geschlechterunterschied in den jüngeren Altersgruppen, was durch eine höhere materielle Werteorientierung in diesem Alter erklärbar sein könnte [Dittmar, 2005a]. Einheitlich zeigt sich, dass jüngere Menschen vermehrt zu pathologischem Kaufen tendieren [Maraz et al., 2016]. Weitere soziodemografische Einflüsse auf pathologisches Kaufen, wie z.B. das Einkommen, die Schulbildung oder der Partnerschaftsstatus, konnten bisher weder klinisch noch epidemiologisch nachgewiesen werden [Hubert et al., 2014; Müller et al., 2016]. Folglich handelt es sich um eine Störung, die weniger spezifische Risikogruppen als vielmehr die Allgemeinbevölkerung betrifft und die ganz offensichtlich ein PublicHealth-Problem darstellt.

\section{Behandlung}

Bis heute liegen nur wenige evaluierte Behandlungskonzepte für pathologisches Kaufen vor [Hague et al., 2016; Soares et al., 2016]. Am besten beschrieben sind kognitiv-verhaltenstherapeutische Ansätze [Benson et al., 2014; Granero et al., 2017; Müller et al., 2018]. In 3 kontrollierten Psychotherapiestudien [Mitchell et al., 2006; Müller et al., 2008; Müller et al., 2013] wurde die Wirksamkeit eines in den USA entwickelten kognitiv-behavioralen Gruppentherapiemanuals [Mitchell et al., 2006], das für den deutschen Sprachraum modifiziert wurde [Müller und de Zwaan, 2008], überprüft. Eine rezente praxisorientierte Beschreibung der kognitiven Verhaltenstherapie bei Patienten mit pathologischem Kaufen findet sich bei Müller et al. [2018].

Im Gegensatz zur nachgewiesenen Wirksamkeit der kognitiven Verhaltenstherapie steckt die Studienlage zur medikamentösen Behandlung von pathologischem Kaufen noch in den Kinderschuhen [Soares et al., 2016]. Bislang publiziert wurden mehrere Kasuistiken mit dem Opioid-Rezeptorantagonisten Naltrexon sowie einige offene und kontrollierte Fallserien mit selektiven Serotonin-Wiederaufnahmehemmern (selective serotonin reuptake inhibitors; SSRIs) (z.B. Fluvoxamin, Citalopram, Escitalopram; Übersicht bei Soares et al. [2016]). Für Letztere konnte keine Überlegenheit gegenüber Placebo nachgewiesen werden. Limitierend waren zudem die teilweise sehr hohen Abbruchquoten (50\%) und die geringen Fallzahlen. In den letzten Jahren wurden daneben noch einige Fallbeispiele mit Memantine und Buproprionaugmentation veröffentlicht [Soares et al., 2016]. Systematische Medikamentenstudien stehen jedoch aus. 


\section{Klassifikation}

\section{Pathologisches Kaufen als Zwangsstörung}

Ausgehend von einigen phänomenologischen Ähnlichkeiten mit Zwängen wurde pathologisches Kaufen in den 1990er Jahren zunächst als spezifische Form einer Zwangsstörung diskutiert [Lawrence et al., 2014]. In diese Zeit fallen auch die bereits erwähnten offenen, Erfolg versprechenden Medikamentenstudien mit SSRIs, die bekanntlich in der Behandlung von Zwangserkrankungen zugelassen sind. Die Ergebnisse dieser offenen Studien ließen sich jedoch nicht durch kontrollierte Studien bestätigen (Übersicht bei Soares et al. [2016]).

Hinzu kamen frühere Befunde hinsichtlich einer hohen Komorbidität von pathologischem Kaufen mit Zwangsstörungen. Allerdings haben vor dem Erscheinen des DSM-5 [APA, 2015], in dem pathologisches Horten erstmals als eigenständige Erkrankung gelistet wurde, viele Untersuchungen nicht zwischen Zwangsstörungen und pathologischem Horten unterschieden. Es kann davon ausgegangen werden, dass die früher berichtete hohe Komorbidität von pathologischem Kaufen und Zwangserkrankungen am ehesten auf die hohe Komorbidität von pathologischem Kaufen mit pathologischem Horten zurückzuführen ist [Torres et al., 2016].

Phänomenologisch stellt die anhaltende gedankliche Beschäftigung mit Kaufen (preoccupation) tatsächlich ein Kernsymptom von pathologischem Kaufen dar. Kontrastierend zu Zwangsstörungen handelt es sich bei der gedanklichen Beschäftigung im Rahmen von pathologischem Kaufen nicht um ego-dystone, intrusive Gedanken, sondern vielmehr um ego-syntone, identitätsstiftende Kognitionen und materiell orientierte Werteeinstellungen [Moulding et al., 2017]. Wichtig ist weiterhin, dass das Kaufen zumindest im Anfangsstadium der Erkrankung eine belohnungssuchende, impulsive Handlung zur schnellen Befriedigung eines drängenden Besitzwunschs darstellt [Kellett und Bolton, 2009]. Erst im Verlauf entwickeln sich habituelle, zunehmend kompulsive Kaufmuster, die der Kompensation von Diskomfort und negativen Befindlichkeiten dienen (negative Verstärkungsprozesse). In klinischen Studien konnte gezeigt werden, dass pathologisches Kaufen phänotypisch mehr Ähnlichkeiten mit anderen Verhaltenssüchten (z.B. mit der Glücksspielstörung) als mit Zwangsstörungen aufweist [Lawrence et al., 2014; Mestre-Bach et al., 2017].

Im DSM-5 [APA, 2015] wird «excessive buying» als Beispiel für «excessive acquisition» im gleichnamigen Specifier der «hoarding disorder» aufgelistet. Laut Release-Version der ICD-11 [WHO, 2018] zählt «excessive acquisition» (ICD-11: «repetitive urges or behaviours related to amassing or buying items») zu den diagnostischen Kriterien von pathologischem Horten [Fontenelle und Grant, 2014]. Entsprechend ließe sich argumentieren, dass pathologisches Kaufen lediglich ein Symptom von pathologischem Horten ist. Dagegen spricht jedoch, dass zumindest in klinischen Stichproben nur etwa die Hälfte der Patienten mit pathologischem Kaufen auch unter pathologischem Horten litten, während die übrigen Patienten keine Probleme damit hatten, sich von den gekauften Gegenständen zu trennen, und entsprechend auch nicht über Unordnung aufgrund angesammelter Konsumgüter klagten [Möllen- kamp et al., 2015]. An dieser Stelle muss erwähnt werden, dass Patienten mit pathologischem Kaufen und komorbidem pathologischen Horten in Psychotherapiestudien eine geringere TherapieResponse gezeigt haben als Patienten ohne die Begleiterkrankung [Müller et al., 2008]. Insgesamt sollte erwogen werden, pathologisches Horten als Specifier von pathologischem Kaufen zu definieren. Dies könnte auch bei der Entwicklung und Überprüfung differenzieller Therapiekonzepte hilfreich sein.

\section{Pathologisches Kaufen als Impulskontrollstörung}

In den letzten 20 Jahren wurde die Klassifikation von pathologischem Kaufen als Impulskontrollstörung favorisiert [Grant und Chamberlain, 2016; Müller et al., 2015a]. Wie bereits erwähnt, handelt es sich zu Beginn der Erkrankung um ein vorwiegend impulsives, belohnungssuchendes Verhalten. Ausgehend von Patientenberichten scheinen die unangemessenen Kaufepisoden durch einen Spannungsbogen mit vorausgehender Anspannung vor und Entspannung sowie Befriedigung nach dem Kaufakt gekennzeichnet zu sein. Zu den Charakteristika von pathologischem Kaufen zählen durchaus die hohe Impulsivität der Patienten und der wiederholte Verlust der Kontrolle über den Warenkonsum, was in manchen Fällen mit der Verletzung der Rechte Dritter (z.B. Eingehungsbetrug) verbunden ist. Die genannten Charakteristika sind typisch für Störungen der Impulskontrolle [WHO, 2018]. Im aktuellen ICD-11-Coding-Tool wird die «compulsive buying-shopping disorder» entsprechend als Beispiel in der Residualkategorie (6C7Y) «Other specified impulse control disorders» genannt [WHO, 2018]. Dieser Vorschlag ist zu begrüßen, weil pathologisches Kaufen damit erstmals Erwähnung im ICD-Coding-Tool findet. Gleichzeitig ist diese Einordnung angesichts aktueller Forschungsbefunde bezüglich Ähnlichkeiten zwischen pathologischem Kaufen und süchtigen Verhaltensweisen kritisch zu sehen, worauf im Folgenden näher eingegangen wird.

\section{Pathologisches Kaufen als Verhaltenssucht}

An dieser Stelle werden Überlegungen zusammengefasst, die die nosologische Einordnung von pathologischem Kaufen als «Störung in Zusammenhang mit suchtartigem Verhalten» und damit als Kandidat für die ICD-11-Kategorie (6C5Y) «Other specified disorders due to addictive behaviours» [WHO, 2018] implizieren [Müller et al., in press]. Offensichtlich bestehen Ähnlichkeiten zwischen den zugrundeliegenden kognitiven und neurobiologischen Mechanismen von pathologischem Kaufen, Glücksspielstörung, Internetspielstörung und Substanzgebrauchsstörungen. Dafür sprechen neuere experimentelle Befunde, die im Einklang stehen mit der Incentive-Sensitization-Theorie zur Entstehung süchtigen Verhaltens von Berridge und Robinson [2016]. Dieser psychobiologische Ansatz geht davon aus, dass der Substanzkonsum initial hedonistisch motiviert ist (liking). Die wiederholte Substanzeinnahme geht mit einer Sensitivierung des dopaminergen Belohnungssystems (vor allem des Nucleus accumbens) einher, was in einer «incentive salience» (Anreizhervorhebung) suchtbezogener Hinweisreize resultiert. Letztere werden nun als besonders attraktiv wahrgenommen und lenken die Aufmerksamkeit stärker auf sich als andere Reize. 


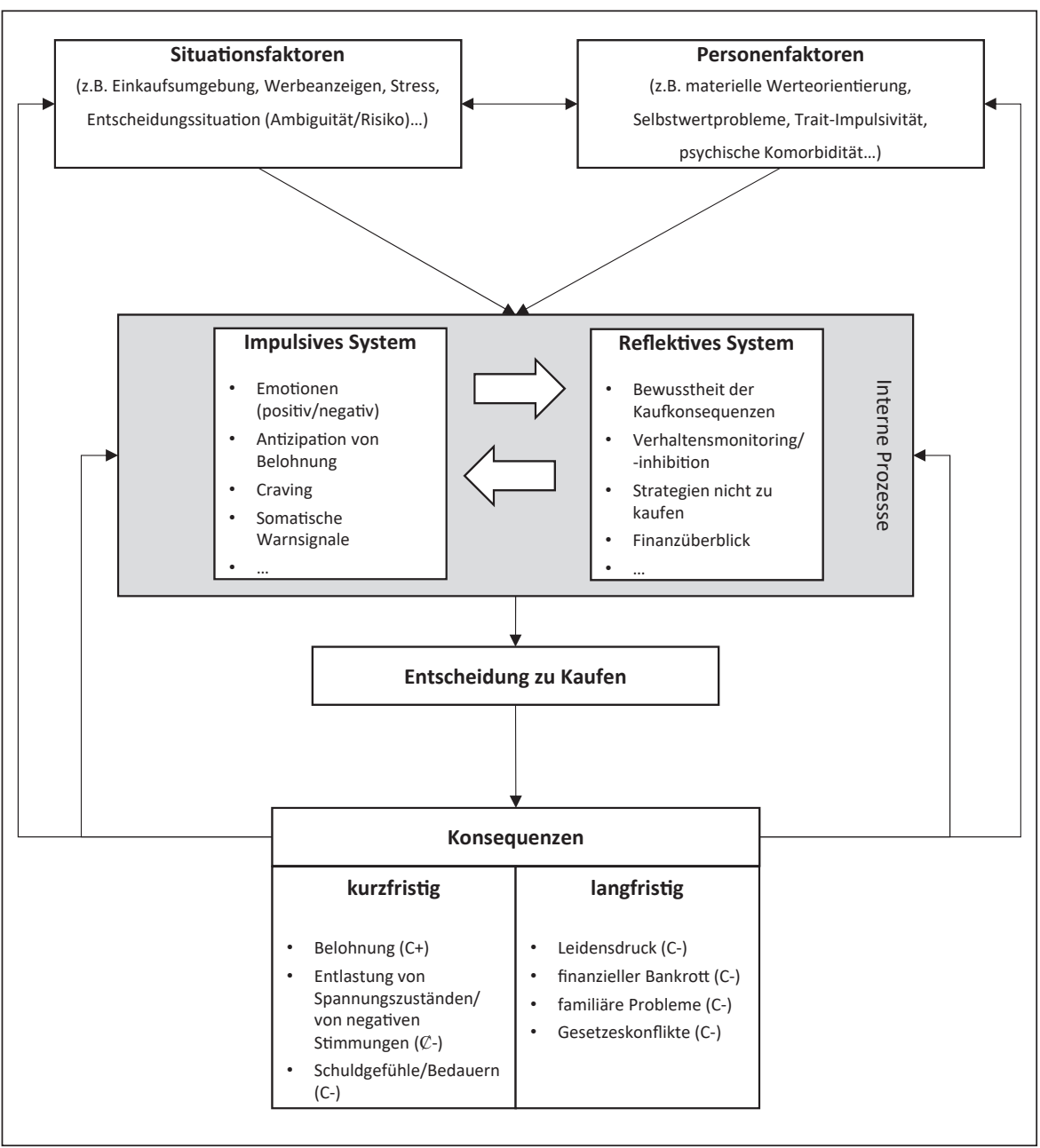

Abb. 1. Ätiologiemodell für pathologisches Kaufen nach Trotzke et al. [2017].

Die ursprünglich angenehme Wirkung der Droge lässt mit der Zeit nach. Allerdings lösen die suchtbezogenen Hinweisreize nun ihrerseits vermehrt konditionierte Reaktionen aus, was sich in starkem Craving (wanting) äußert [Berridge und Robinson, 2016].

Als Hinweise für die prominente Rolle dopaminerger Bahnen des mesolimbischen Systems beim pathologischen Kaufen können die bereits erwähnten Kasuistiken zum erfolgreichen Einsatz des Opioid-Rezeptorantagonisten Naltrexon gelten, wobei noch keine systematischen Medikamentenstudien zum Einsatz von OpioidRezeptorantagonisten vorliegen [Soares et al., 2016]. Überzeugender erscheinen die Ergebnisse der in den letzten 5 Jahren erschienenen experimentellen Studien. Diese wiesen bei Menschen mit pathologischem Kaufen ein unvorteilhaftes Entscheidungsverhalten, einen hohen Anreizwert (incentive salience) von visuellen Kaufreizen, eine längerfristige Hypersensibilität gegenüber visuellen Kaufreizen und die Ausbildung von Craving sowie habituellen Reiz-Reaktions-Assoziationen nach [Mestre-Bach et al., 2017; Trotzke et al., 2017; Vogel et al., 2018; Voth et al., 2014].

Im Hinblick auf diese empirischen Befunde und die daraus resultierende Nähe von pathologischem Kaufen zu Suchterkrankungen wurde unlängst ein Ätiologiemodell für pathologisches Kaufen vorgeschlagen, das auf Modellvorstellungen für internetbezogene Suchterkrankungen [Brand et al., 2016] sowie auf Dual-Prozess-Theorien des Entscheidungsverhaltens [Schiebener und Brand, 2015] basiert. Nach Trotzke et al. [2017] wird die Kaufentscheidung bei Menschen mit pathologischem Kaufen durch ein überaktives, neuronal repräsentiertes impulsives System getriggert (z.B. in Form von belohnungsantizipierendem Craving oder internalen Zuständen). Kontroll- und Inhibitionsprozesse im Sinne eines präfrontal repräsentierten reflektiven Systems werden hingegen unterdrückt. Verschiedene Situations- und Personenfaktoren sowie die kurz- und langfristigen Konsequenzen nach dem Kaufen haben einen modulierenden Einfluss auf die beiden interagierenden Systeme (impulsiv vs. reflektiv; Trotzke et al. [2017]). Abbildung 1 bietet eine grafische Darstellung des Modells und der postulierten Zusammenhänge.

Zum Verständnis von pathologischem Kaufen tragen im Übrigen auch Untersuchungen aus der neurowissenschaftlichen Konsumentenverhaltensforschung (Neuroökonomik) bei, die sich nicht nur mit Markenwirkung, sondern auch mit neuralen Mechanismen von Kaufentscheidungen befassen. Obgleich die meisten konsumentenpsychologischen Studien auf eine ausführliche klinische Diagnostik (inklusive Komorbidität) verzichten und die Kaufsuchtneigung der untersuchten Teilnehmenden lediglich mittels kaufsuchtspezifischer Fragebogen-Scores charakterisieren, liefern sie wertvolle Hinweise zum Verständnis der neurokognitiven Prozesse beim pathologischen Kaufen. Beispielsweise ergab eine aktuelle Untersuchung zur Infor- 
mationsverarbeitung und visuellen Aufmerksamkeit bei Personen mit einer starken Neigung zu pathologischem Kaufen (gemessen mit dem SKSK; Raab et al. [2005]) suchttypische Aufmerksamkeitsverzerrungen (attentional bias) [Serfas et al., 2016].

Die bislang einzige Bildgebungsstudie mit Patienten stammt gleichfalls aus der Neuroökonomik. In Analogie zu Substanzkonsumstörungen deuten die Befunde dieser kontrollierten Studie mit funktioneller Magnetresonanztomografie auf eine erhöhte Belohnungssensitivität und ein verringertes Verlustempfinden beim pathologischen Kaufen hin [Raab et al., 2011]. Die Präsentation von Konsumgütern, deren Kauf die Patienten antizipierten, ging mit einer Aktivierung im Nucleus accumbens einher. Hingegen zeigte sich eine verringerte Aktivität der Insula während der Präsentation des Warenpreises dieser Produkte [Raab et al., 2011].

\section{Fazit}

Beim pathologischen Kaufen handelt es sich um eine vermutlich häufige Psychopathologie, die mit negativen Konsequenzen für die Betroffenen assoziiert ist und deren Anerkennung als psychische Erkrankung längst überfällig ist. Aktuelle klinische und experimentelle Studien legen aufgrund der Ähnlichkeiten von patho- logischem Kaufen und Substanzkonsumstörungen sowie der Glücksspielstörung die Klassifikation als «Störung in Zusammenhang mit suchtartigem Verhalten» (ICD-11: «disorder due to addictive behaviours») nahe. Diese Studienergebnisse sollten mit größeren Stichproben repliziert werden. Um die Mechanismen von pathologischem Kaufen aufzudecken, sollte die Forschung außerdem auf die Entwicklung einer einheitlichen Diagnostik auf der Basis evaluierter diagnostischer Kriterien, auf die Durchführung von interviewbasierten Prävalenz- und Verlaufsuntersuchungen und auf weitere grundlagenorientierte Studien fokussieren. Dies würde zur empirisch fundierten Ableitung von Behandlungskonzepten beitragen.

\section{Disclosure Statement}

Die Autoren erklären, dass keine Interessenkonflikte bestehen.

\section{Danksagung}

Diese Arbeit entstand im Rahmen des vom Deutschen Akademischen Austauschdienst (DAAD) geförderten Projekts «Neue Erhebungsmethoden und diagnostische Kriterien für pathologisches Kaufen» (Projektnummer 57387119).

\section{Literatur}

APA (American Psychiatric Association): Diagnostisches und Statistisches Manual Psychischer Störungen DSM5. Göttingen, Hogrefe, 2015.

Benson AL, Eisenach D, Abrams L, van Stolk-Cooke K: Stopping overshopping: a preliminary randomized controlled trial of group therapy for compulsive buying disorder. Journal of Groups in Addiction \& Recovery 2014;9:97-125.

Berridge KC, Robinson TE: Liking, wanting, and the incentive-sensitization theory of addiction. Am Psychol 2016;71:670-679.

Black DW, Shaw M, Allen J: Five-year follow-up of people diagnosed with compulsive shopping disorder. Compr Psychiatry 2016;68:97-102.

Bleuler E: Lehrbuch der Psychiatrie, ed 4. Berlin, Verlag von Julius Springer, 1923.

Brand M, Young KS, Laier C, Wölfling K, Potenza MN: Integrating psychological and neurobiological considerations regarding the development and maintenance of specific Internet-use disorders: an interaction of Person-Affect-Cognition-Execution (I-PACE) model. Neurosci Biobehav Rev 2016;71:252-266.

Claes L, Müller A: Resisting temptation: Is compulsive buying an expression of personality deficits? Curr Addict Rep 2017;4:237-245.

Coley A, Burgess B: Gender differences in cognitive and affective impulse buying. Journal of Fashion Marketing and Management 2003;7;282-295.

Dittmar H: Compulsive buying - a growing concern? An examination of gender, age, and endorsement of materialistic values as predictors. Br J Psychol 2005a;96(Pt 4):467-491.

Dittmar H: A new look at "compulsive buying»: self-discrepancies and materialistic values as predictors of compulsive buying tendency. J Soc Clin Psychol 2005b;24:832-859.
Dittmar H, Long K, Bond R: When a better self is only a button click away: associations between materialistic values, emotional and identity-related buying motives, and compulsive buying tendency online. J Soc Clin Psychol 2007;26:334-361.

Faber RJ, O'Guinn TC: A clinical screener for compulsive buying. J Consum Res 1992;19:459-469.

First MB, Williams JBW, Spitzer RL, Gibbon M: Structured Clinical Interview for DSM-IV-TR Axis I Disorders: Clinical Trials Version (SCID-CT). New York, Biometrics Research, 2007.

Fontenelle LF, Grant JE: Hoarding disorder: a new diagnostic category in ICD-11? Rev Bras Psiquiatr 2014; 36(suppl 1):28-39.

Granero R, Fernández-Aranda F, Mestre-Bach G, Steward T, Baño M, et al: Cognitive behavioral therapy for compulsive buying behavior: predictors of treatment outcome. Eur Psychiatry 2017;39:57-65.

Granero R, Fernández-Aranda F, Steward T, Mestre-Bach G, Baño M, et al: Compulsive buying behavior: characteristics of comorbidity with gambling disorder. Front Psychol 2016;7:625.

Grant JE, Chamberlain SR: Expanding the definition of addiction: DSM-5 vs. ICD-11. CNS Spectr 2016;21:300303.

Hague B, Hall J, Kellett S: Treatments for compulsive buying: a systematic review of the quality, effectiveness and progression of the outcome evidence. J Behav Addict 2016;5,379-394.

Hubert M, Hubert M, Gwozdz W, Raab G, Reisch L: Compulsive buying: An increasing problem? Investigating and comparing trends in Germany and Denmark, 2010-2012. J Verbrauch Lebensm 2014;9:280-284.

Kellett S, Bolton JV: Compulsive buying: a cognitive-behavioural model. Clin Psychol Psychother 2009;16:83-99.

Kraepelin E: Psychiatrie - Ein Lehrbuch für Studierende und Ärzte, ed 8. Leipzig, Verlag Johann Ambrosius Barth, 1909.
Kyrios M, McQueen P, Moulding R: Experimental analysis of the relationship between depressed mood and compulsive buying. J Behav Ther Exp Psychiatry 2013;44: 194-200.

Lawrence LM, Ciorciari J, Kyrios M: Relationships that compulsive buying has with addiction, obsessive-compulsiveness, hoarding, and depression. Compr Psychiatry 2014;55:1137-1145.

Maraz A, Griffiths MD, Demetrovics Z: The prevalence of compulsive buying: A meta-analysis. Addiction 2016; 111:408-419.

McElroy SL, Keck PE Jr, Pope HG Jr, Smith JM, Strakowski SM: Compulsive buying: a report of 20 cases. J Clin Psychiatry 1994;55:242-248.

Mestre-Bach G, Steward T, Jiménez-Murcia S, FernándezAranda F: Differences and similarities between compulsive buying and other addictive behaviors. Curr Addict Rep 2017;4:228-236.

Miltenberger RG, Redlin J, Crosby R, Stickney M, Mitchell $\mathrm{J}$, et al: Direct and retrospective assessment of factors contributing to compulsive buying. J Behav Ther Exp Psychiatry 2003;34:1-9.

Mitchell JE, Burgard M, Faber R, Crosby RD, de Zwaan M: Cognitive behavioral therapy for compulsive buying disorder. Behav Res Ther 2006;44:1859-1865.

Möllenkamp M, de Zwaan M, Müller A: Hoarding with and without excessive buying: results of a pilot study. Psychopathology 2015;48:56-59.

Monahan P, Black DW, Gabel J: Reliability and validity of a scale to measure change in persons with compulsive buying. Psychiatry Res 1996;64:59-67.

Moulding R, Duong A, Nedeljkovic M, Kyrios M: Do you think that money can buy happiness? A review of the role of mood, materialism, self, and cognitions in compulsive buying. Curr Addict Rep 2017;4:254-261. 
Müller A, Arikian A, de Zwaan M, Mitchell JE: Cognitivebehavioural group therapy versus guided self-help for compulsive buying disorder: a preliminary study. Clin Psychol Psychother 2013;20:28-35.

Müller A, Claes L, Demetrovics Z, de Zwaan M, Fernandes-Aranda F, et al: Buying-shopping disorder - is there enough evidence to support its inclusion in ICD11 ? CNS Spectrums; in press.

Müller A, de Zwaan M: Therapieansätze bei pathologischem Kaufen (Treatment of compulsive buying) Fortschr Neurol Psychiatr 2008;76:478-483.

Müller A, de Zwaan M, Mitchell JE, Zimmermann T: Pathological buying and partnership status. Psychiatry Res 2016;239:122-123.

Müller A, Mitchell JE, Crosby RD, Cao L, Johnson J, et al Mood states preceding and following compulsive buying episodes: an ecological momentary assessment study. Psychiatry Res 2012;200:575-580.

Müller A, Mitchell JE, Crosby RD, Gefeller O, Faber RJ, et al: Estimated prevalence of compulsive buying in Germany and its association with sociodemographic characteristics and depressive symptoms. Psychiatry Res 2010;180:137-142.

Müller A, Mitchell JE, de Zwaan M: Compulsive buying. Am J Addict 2015a;24:132-137.

Müller A, Mitchell JE, Vogel B, de Zwaan M: New assessment tools for buying disorder. Curr Addict Rep 2017; 4:221-227.

-Müller A, Müller U, Albert P, Mertens C, Silbermann A, et al: Hoarding in a compulsive buying sample. Behav Res Ther 2007;45:2754-2763.

Müller A, Müller U, Silbermann A, Reinecker H, Bleich S, et al: A Randomized, controlled trial of group cognitive-behavioral therapy for compulsive buying disorder: posttreatment and 6-month follow-up results. J Clin Psychiatry 2008;69:1131-1138.
Müller A, Trotzke P, Mitchell JE, de Zwaan M, Brand M: The Pathological Buying Screener: development and psychometric properties of a new screening instrument for the assessment of pathological buying symptoms. PLoS One 2015b;10:e0141094.

Müller A, Wölfling K, Müller KW: Verhaltenssüchte - Pathologisches Kaufen, Spielsucht und Internetsucht. Göttingen, Hogrefe, 2018.

Neuner M, Raab G, Reisch LA: Compulsive buying in maturing consumer societies: An empirical re-inquiry. J Econ Psychol 2005;26:509-522.

Raab G, Elger CE, Neuner M, Weber B: A neurological study of compulsive buying behaviour. J Consum Policy 2011;34:401-413.

Raab G, Neuner M, Reisch LA, Scherhorn G: SKSK Screeningverfahren zur Erhebung von kompensatorischem und süchtigem Kaufverhalten. Ein Testmanual. Göttingen, Hogrefe, 2005.

Scherhorn G, Reisch LA, Raab G: Addictive buying in West Germany: an empirical study. J Consum Policy 1990; 13:355-387.

Schiebener J, Brand M: Decision making under objective risk conditions - a review of cognitive and emotional correlates, strategies, feedback processing, and external influences. Neuropsychol Rev 2015;25:171-198.

Serfas BG, Büttner OB, Florack A: Using implementation intentions in shopping situations: how arousal can help shield consumers against temptation. Appl Cogn Psychol 2016;30:672-680.

Soares C, Fernandes N, Morgado P: A review of pharmacologic treatment for compulsive buying disorder. CNS Drugs 2016;30:281-291.
Torres AR, Fontenelle LF, Shavitt RG, Ferrão YA, do Rosário MC, et al: Comorbidity variation in patients with obsessive-compulsive disorder according to symptom dimensions: results from a large multicentre clinical sample. J Affect Disord 2016;190:508-516.

Trotzke P, Brand M, Starcke K: Cue-reactivity, craving, and decision making in buying disorder: a review of the current knowledge and future directions. Curr Addict Rep 2017;4:246-253.

Trotzke P, Starcke K, Müller A, Brand M: Pathological buying online as a specific form of Internet addiction: a model-based experimental investigation. PLoS One 2015;10:e0140296.

Vogel V, Kollei I, Duka T, Snagowski J, Brand M, et al: Pavlovian-to-instrumental transfer: a new paradigm to assess pathological mechanisms with regard to the use of Internet applications. Behav Brain Res 2018;347:816.

Voth EM, Claes L, Georgiadou E, Selle J, Trotzke P, et al: Reactive and regulative temperament in patients with compulsive buying and non-clinical controls measured by self-report and performance-based tasks. Compr Psychiatry 2014;55:1505-1512.

WHO (World Health Organization): International Classification of Diseases 11th Revision, 2018. https://icd. who.int/ct11_2018/icd11_mms/en/release\#/ (letzter Zugriff 31. August 2018).

Williams AD: Quality of life and psychiatric work impairment in compulsive buying: increased symptom severity as a function of acquisition behaviors. Compr Psychiatry 2012;53:822-828.

Zhang C, Brook JS, Leukefeld CG, De La Rosa M, Brook DW: Compulsive buying and quality of life: an estimate of the monetary cost of compulsive buying among adults in early midlife. Psychiatry Res 2017;252: 208-214. 
DuEPublico

Duisburg-Essen Publications online
UNIVERSITÃT
DE SISSBEN R G

offen im Denken

Ub $\begin{aligned} & \text { universitäts } \\ & \text { bibliothek }\end{aligned}$

Dieser Text wird via DuEPublico, dem Dokumenten- und Publikationsserver der Universität Duisburg-Essen, zur Verfügung gestellt. Die hier veröffentlichte Version der E-Publikation kann von einer eventuell ebenfalls veröffentlichten Verlagsversion abweichen.

DOI: $\quad 10.1159 / 000493888$

URN： urn:nbn:de:hbz:464-20210506-091920-7

Dieser Beitrag ist mit Zustimmung des Rechteinhabers aufgrund einer (DFGgeförderten) Allianz- bzw. Nationallizenz frei zugänglich. 